\title{
Long-term reliability of the telemetric Neurovent-P-tel sensor: in vivo case report
}

\author{
Jorge Tirado-Caballero, MD,1,3 Andres Muñoz-Nuñez, MD, PhD, 1,3 Santiago Rocha-Romero, MD,1,3 \\ Mónica Rivero-Garvía, MD, PhD, , Emilio Gomez-González, BS, $\mathrm{PhD},{ }^{2,3}$ and \\ Javier Marquez-Rivas, MD, PhD ${ }^{1,3}$
}

\begin{abstract}
${ }^{1}$ Neurosurgery Service, Virgen del Rocío University Hospital, Seville; ${ }^{2}$ Group of Interdisciplinary Physics, University of Seville; and ${ }^{3}$ Group of Applied Neuroscience, Biomedicine Institute of Seville, Spain

Intracranial pressure (ICP) measurements are imperative for the proper diagnosis and treatment of several neurological disorders. Telemetric sensors have shown their utility for ICP estimation in short-term monitoring in humans. However, their long-term reliability is uncertain. The authors present the case of a 37-year-old woman diagnosed with benign intracranial hypertension and obesity. The patient underwent gastric bypass surgery for ICP control. In order to monitor ICP before and after bariatric surgery, a Neurovent-P-tel sensor was implanted in the left frontal lobe. After gastric bypass, normal ICP values were recorded, and the patient's visual fields improved. However, the patient experienced incapacitating daily headaches. The authors decided to implant a Codman Microsensor ICP transducer in the right frontal lobe to assess the long-term reliability of the Neurovent-P-tel measurements. A comparison of the recordings at 24 and 48 hours showed good correlation and reliability during long-term monitoring with the Neurovent-P-tel, with minimal zero drift after 11 months of implantation.
\end{abstract}

https://thejns.org/doi/abs/10.3171/2018.4.JNS172988

KEYWORDS intracranial pressure; Neurovent-P-tel; telemetric monitoring; diagnostic technique

I NTRACRANIAL pressure (ICP) monitoring is essential for the optimal diagnosis and treatment of several neurological and neurosurgical disorders. ${ }^{11,13}$ Lumbar punctures or intracranial wired transducers have been used for this purpose. However, the risk of infection due to repetitive invasive procedures in the case of lumbar punctures or extracranial exposure of the device and zero drift after 6-7 days of implantation in the case of nontelemetric sensors make these methods useless for long-term ICP monitoring.

Telemetric sensors may be employed to solve this problem through an intracranial implanted device. Several devices have been used for this purpose, such as the Rotterdam transducer ${ }^{5}$ and the Cosman telemetric sensor. ${ }^{4}$ However, their implantation in routine clinical practice has been limited to date. In recent years, the Neurovent-Ptel (Raumedic), a micro-strain gauge telemetric sensor, has shown good clinical results and contributed to the diagnosis of many neurological diseases, such as hydrocephalus, benign intracranial hypertension $(\mathrm{BIH})$, and valve overdrainage or underdrainage. ${ }^{2}$

Patients affected by complex hydrocephalus, syndromic craniosynostosis, ${ }^{10}$ or uncertainty regarding correct CSF dynamics may also benefit from devices that provide neurosurgeons with ICP measurements over a prolonged period of time without additional invasive procedures. The Neurovent P-tel sensor has shown short-term reliability for ICP monitoring in humans, and it has received the $\mathrm{CE}$ mark for a maximum period of 29 days. It also has shown good reliability for long-term ICP measurements in animals, ${ }^{14}$ but its zero drift and reliability in long-term ICP monitoring in the human brain is still unknown.

We present the case of a 37-year-old woman who was diagnosed with $\mathrm{BIH}$ and initially monitored with a Neurovent P-tel sensor for 11 months. At that time, an additional Codman Microsensor ICP transducer was implanted in the contralateral frontal lobe. A comparison between mea- 


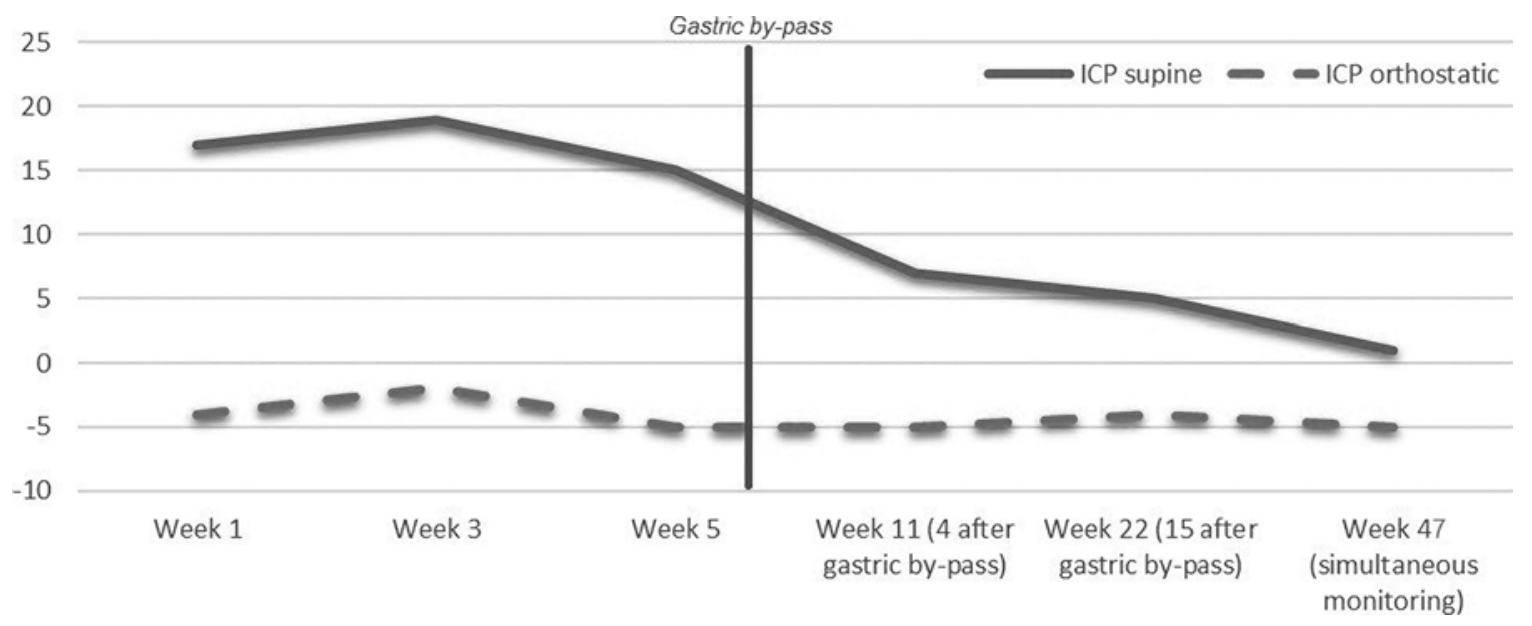

FIG. 1. Raumedic Neurovent-P-tel measurements. Measurements were performed during weeks 1,3 , and 5 after Neurovent-Ptel implantation. Previous to gastric bypass surgery, ICP in the supine position was very high, i.e., over $15 \mathrm{~mm} \mathrm{Hg}$. After gastric bypass surgery, an improvement in ICP was seen with a decrease to within the reference range.

surements was done after 24 and 48 hours of simultaneous ICP monitoring.

\section{Case Report}

This 37-year-old woman had a history of smoking, arterial hypertension, fibromyalgia, gestational diabetes, and morbid obesity. She was experiencing chronic headaches and ongoing loss of visual acuity. Visual field campimetry results indicated increasing visual impairment, and funduscopy showed optic nerve edema. The patient's headache pattern was not associated with an orthostatic or supine position, and MR and CT scans did not show any pathological entities. With a suspicion of benign ICP hypertension, a lumbar puncture was performed and revealed high ICP $(38 \mathrm{~mm} \mathrm{Hg})$. The patient was placed on a regimen of pharmacological treatment with oral acetazolamide $(250$ mg every 8 hours) and oral topiramate (50 mg every 12 hours). Four months later, the patient showed progressive worsening of visual acuity and incapacitating headaches.

Another lumbar puncture was performed and again revealed very high ICP $(29 \mathrm{~mm} \mathrm{Hg})$. Due to the patient's

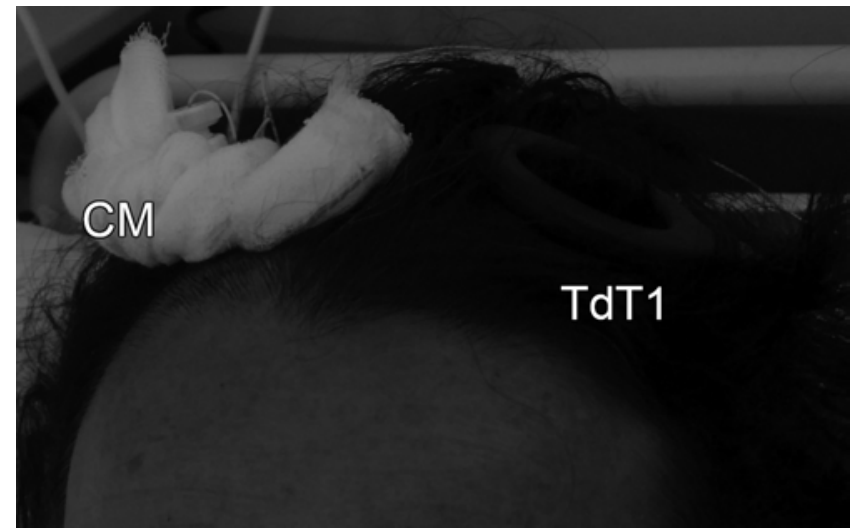

FIG. 2. Reader TDT1 in the left frontal lobe position reading ICP from the Raumedic Neurovent-P-tel. The Codman Microsensor ICP transducer $(\mathrm{CM})$ is located in the right frontal lobe . morbid obesity, bariatric surgery was proposed as a firstline treatment for ICP control. ${ }^{7,15}$ In order to monitor ICP before and after surgery, we decided to implant an intraparenchymal Neurovent-P-tel sensor for long-term ICP registration.

Under sedation and local anesthesia, a semicircular left frontal incision was done. The cranial calvaria was exposed, and the sensor was implanted through a frontal burr hole and a small dural incision. The skin was sutured with polypropylene stitches.

Measurements were done in both the orthostatic and supine positions. Three measurements were taken every 2 weeks before gastric bypass surgery and revealed high ICP values. After bariatric surgery, the patient lost $39 \mathrm{~kg}$, and her ICP returned to normal physiological levels (Fig. 1). Visual field campimetry showed minimal visual improvement, and funduscopy showed resolution of the optic nerve edema. However, the patient reported progressively worsening headaches that were not controlled by pharmacological therapy. These headaches were not position dependent, and the patient reported acute episodes of sonophobia and photophobia.

After 11 months of follow-up with daily headaches, we decided to check and confirm the long-term reliability of the telemetric sensor by implanting a contralateral intraparenchymal Codman Microsensor ICP transducer connected to PowerLab 4/35 (ADInstruments). Continuous monitoring was performed using LabChart (v8.1.9, ADInstruments). With the patient positioned at a $45^{\circ}$ angle, Neurovent-P-tel sensor measurements were obtained simultaneously 24 and 48 hours after microsensor implantation (Fig. 2), and Valsalva maneuvers were done during recording to check the ability of both sensors to adapt to changes in ICP. The ICP measurements from both sensors were within the reference range at every time point, and no changes in registration were correlated between ICP and headaches.

A very acceptable correlation was observed between the recordings. A difference of only 3-4 $\mathrm{mm} \mathrm{Hg}$ was seen in the baseline ICP line between the telemetric sensor and 

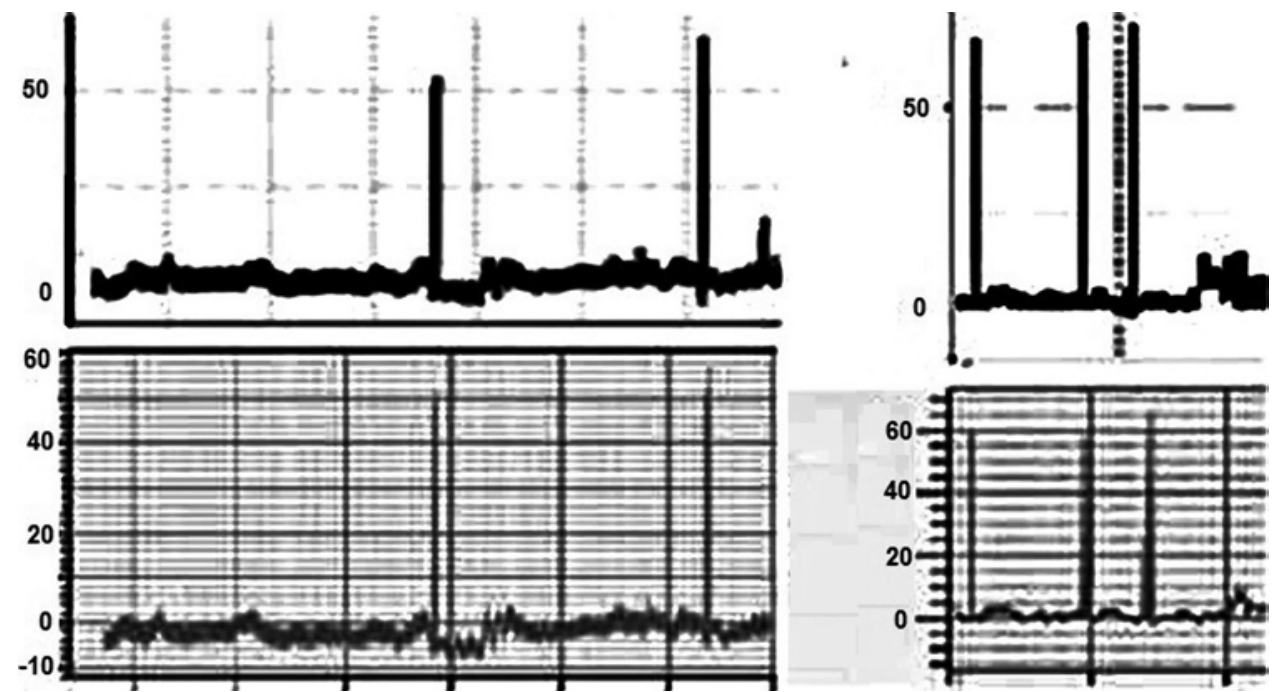

FIG. 3. Comparison between ICP recordings 24 hours (left) and 48 hours (right) after microsensor implantation. Upper: Codman Microsensor ICP measurements. Lower: Raumedic Neurovent-P-tel ICP measurements. Reliable data were observed. The basal ICP line shows minimal differences of 3-4 mm Hg. Valsalva maneuvers were done, showing the ability of both sensors to adapt at an increase and immediate fall in ICP.

the intraparenchymal standard microsensor. When Valsalva maneuvers were done, the patient's ICP increased up to $50 \mathrm{~mm} \mathrm{Hg}$ for a moment in both records and then fell immediately to baseline values, showing good intracranial compliance and proper functioning of the ICP regulation mechanisms (Fig. 3).

After 3 days of continuous ICP monitoring, we confirmed the absence of pathology in CSF dynamics. Both sensors were removed in the operating room, and the patient was discharged home without any surgical complications.

\section{Discussion}

Our knowledge of CSF dynamics has increased significantly in recent years. ICP measurements provide useful data for the diagnosis, follow-up, and treatment of patients with complex hydrocephalus. At present, external ventricular drainage is considered the gold standard for measuring ICP, but the risk of infection, even under the most demanding protocols, ${ }^{12}$ makes this method unsuitable for long-term recording. Many kinds of nontelemetric sensors have been used for ICP measurements, but their use for long-term monitoring is controversial because of the risk of infection through an intracranial-extracranial device and zero drift, as reported in several articles. ${ }^{1,16}$ Also, most intraparenchymal sensors cannot be re-zeroed while recording.

Telemetric sensors offer a theoretical opportunity to assess CSF dynamics over a prolonged period of time and are able to provide ICP measurements without additional invasive procedures after implantation. Zero-drift deviation is, in this case, of great concern for physicians. Chen et al. reported that zero drift in Codman ICP intraventricular and subdural sensors was approximately $5 \mathrm{~mm} \mathrm{Hg}$ after 10 days of ICP monitoring. ${ }^{3}$ For the Camino intraparenchymal fiberoptic sensor, Gelabert-González et al. reported a zero drift of $7.3 \pm 5.1 \mathrm{~mm} \mathrm{Hg}$ after 7 days of monitoring. ${ }^{6}$ A positive correlation has been found between zero drift and the duration of monitoring. The Neurovent-P-tel had reliable measurements with acceptable zero-drift deviation for 18 months in minipigs, although in that series, the sensors were implanted inside the peritoneal cavity. ${ }^{8}$ Our ICP data recorded using both the Neurovent-P-tel and Codman Microsensor show that zero drift of telemetric sensors in the human brain is similar to that shown in previous series (4-5 mm Hg in a period of 11 months), corresponding to group 2 in the minipig study. This zero drift is practically negligible compared with that of an intraventricular or subdural nontelemetric sensor.

In humans, Lilja et al. showed how useful these telemetric sensors can be for medium-term clinical decisions in a series of 22 patients who were evaluated for more than 3 months. ${ }^{9}$ In this series, one-third of the recording sessions led to surgical revision of the shunt. Magnéli et al. reported the case of a patient with complex craniosynostosis and showed the utility of these sensors over a period of 30 months. ${ }^{10}$ In that case, the transducer showed $1 \mathrm{~mm}$ $\mathrm{Hg}$ of zero drift after device extraction, but no verification of ICP measurements was done during implantation, as in our patient.

In our case, the patient had both BIH and obesity, and we used a Neurovent-P-tel sensor to monitor ICP during clinical follow-up. Since bariatric surgery has shown good clinical results in cases of obesity, ${ }^{7,15}$ we proposed that this patient undergo a gastric bypass procedure. As seen on the recordings, the ICP values normalized several weeks after gastric bypass surgery, and the ICP decreased in the supine position by $7 \mathrm{~mm} \mathrm{Hg}$; there was even a mild improvement in visual acuity. These telemetric measurements were useful in the clinical decision-making, and we did not need to perform additional procedures in this patient, i.e., lumboperitoneal shunt surgery. However, the lack of evidence regarding the long-term reliability of the Neurovent-P-tel 
sensor in recording ICP measurements and the patient's daily headaches motivated us to confirm these values with the implantation of the Codman Microsensor. Our data support the reliability and usefulness of telemetric sensors in making long-term clinical decisions.

\section{Conclusions}

The Neurovent-P-tel sensor is a reliable tool for the long-term measurement of ICP in humans. Zero drift over a prolonged period of ICP monitoring was minimal in our case and showed a very good correlation in the waveform curve analysis.

\section{References}

1. Al-Tamimi YZ, Helmy A, Bavetta S, Price SJ: Assessment of zero drift in the Codman intracranial pressure monitor: a study from 2 neurointensive care units. Neurosurgery 64:94-99, 2009

2. Antes S, Tschan CA, Heckelmann M, Breuskin D, Oertel J: Telemetric intracranial pressure monitoring with the Raumedic Neurovent P-tel. World Neurosurg 91:133-148, 2016

3. Chen L, Du HG, Yin LC, He M, Zhang GJ, Tian Y, et al: Zero drift of intraventricular and subdural intracranial pressure monitoring systems. Chin J Traumatol 16:99-102, 2013

4. Cosman ER, Zervas NT, Chapman PH, Cosman BJ, Arnold MA: A telemetric pressure sensor for ventricular shunt systems. Surg Neurol 11:287-294, 1979

5. de Jong DA, Berfelo MW, de Lange SA, Maas AI: Epidural pressure monitoring with the so-called Rotterdam transducer. Further in vivo results. Acta Neurochir (Wien) 45:301-309, 1979

6. Gelabert-González M, Ginesta-Galan V, Sernamito-García R, Allut AG, Bandin-Diéguez J, Rumbo RM: The Camino intracranial pressure device in clinical practice. Assessment in a 1000 cases. Acta Neurochir (Wien) 148:435-441, 2006

7. Handley JD, Baruah BP, Williams DM, Horner M, Barry J, Stephens JW: Bariatric surgery as a treatment for idiopathic intracranial hypertension: a systematic review. Surg Obes Relat Dis 11:1396-1403, 2015

8. Kiefer M, Antes S, Leonhardt S, Schmitt M, Orakcioglu B, Sakowitz OW, et al: Telemetric ICP measurement with the first CE-approved device: data from animal experiments and initial clinical experiences. Acta Neurochir Suppl (Wien) 114:111-116, 2012

9. Lilja A, Andresen M, Hadi A, Christoffersen D, Juhler M: Clinical experience with telemetric intracranial pressure monitoring in a Danish neurosurgical center. Clin Neurol Neurosurg 120:36-40, 2014

10. Magnéli S, Howells T, Saiepour D, Nowinski D, Enblad P, Nilsson P: Telemetric intracranial pressure monitoring: a noninvasive method to follow up children with complex craniosynostoses. A case report. Childs Nerv Syst 32:13111315,2016

11. Poca M, Sahuquillo J: [Intracranial pressure monitoring and CSF dynamics in patients with neurological disorders: indications and practical considerations.] Neurologia 16:303320, 2001 (Span)

12. Rivero-Garvía M, Márquez-Rivas J, Jiménez-Mejías ME, Neth O, Rueda-Torres AB: Reduction in external ventricular drain infection rate. Impact of a minimal handling protocol and antibiotic-impregnated catheters. Acta Neurochir (Wien) 153:647-651, 2011

13. Rodríguez-Boto G, Rivero-Garvía M, Gutiérrez-González $\mathrm{R}$, Márquez-Rivas J: Basic concepts about brain pathophysiology and intracranial pressure monitoring. Neurologia 30:16-22, 2015

14. Schmitt M, Eymann R, Antes S, Kiefer M: Subdural or intraparenchymal placement of long-term telemetric intracranial pressure measurement devices? Acta Neurochir Suppl 113:109-113, 2012

15. Sugerman HJ, Felton WL III, Sismanis A, Kellum JM, DeMaria EJ, Sugerman EL: Gastric surgery for pseudotumor cerebri associated with severe obesity. Ann Surg 229:634642, 1999

16. Zacchetti L, Magnoni S, Di Corte F, Zanier ER, Stocchetti $\mathrm{N}$ : Accuracy of intracranial pressure monitoring: systematic review and meta-analysis. Crit Care 19:420, 2015

\section{Disclosures}

The authors report no conflict of interest concerning the materials or methods used in this study or the findings specified in this paper.

\section{Author Contributions}

Conception and design: Tirado-Caballero, Muñoz-Nuñez, Marquez-Rivas. Acquisition of data: Tirado-Caballero, RochaRomero. Analysis and interpretation of data: Tirado-Caballero, Gomez-González. Critically revising the article: Muñoz-Nuñez, Rivero-Garvía, Marquez-Rivas. Study supervision: MuñozNuñez, Rivero-Garvía, Gomez-González, Marquez-Rivas.

\section{Correspondence}

Jorge Tirado-Caballero: Virgen del Rocío University Hospital, Sevilla, Spain. jtiradocaballero@gmail.com. 